\title{
Look Beyond the Disease Activity Score of 28 Joints (DAS28): Tender Points Influence the DAS28 in Patients with Rheumatoid Arthritis
}

\author{
EVELIEN TON, MARIJE F. BAKKER, SUZANNE M.M. VERSTAPPEN, EVERT JAN ter BORG, \\ IET A. van ALBADA-KUIPERS, YOLANDE SCHENK, MAAIKE J. van der VEEN, JOHANNES W.J. BIJLSMA, \\ and JOHANNES W.G. JACOBS, on behalf of the Utrecht Rheumatoid Arthritis Cohort Study Group
}

\begin{abstract}
Objective. To explore the influence of tender points (TP) on the Disease Activity Score assessing 28 joints (DAS28) in patients with rheumatoid arthritis (RA).

Methods. In 200 consecutive patients with RA from the outpatient clinic, DAS28 and its components, tender and swollen joint counts (TJC, SJC, respectively), visual analog scale (VAS) for patient's general health (GH), and erythrocyte sedimentation rate (ESR), along with a tender point count (TPC) were assessed. Patients were categorized according to 4 TPC classes: zero, 1-5, 6-10, and $\geq 11$ TP. The influence of TPC classes on DAS28 and its individual components was determined with Kruskal-Wallis tests and correlations between TP and DAS28 and its components were calculated.

Results. In 196 eligible patients, 70\% were female, mean age was 59 years, and median disease duration was 3.9 years; median DAS28 was 3.1; and $49 \%$ had active disease, defined as DAS28 > 3.2. In $15 \%$ of patients, the TPC was $\geq 11$, in $12 \% 6-10$, in $30 \% 1-5$, and in $43 \%$ zero. TPC significantly influenced the DAS2 8 and its less objective components TJC and VAS-GH (i.e., based on patient's report), but not the more objective DAS28 components SJC and ESR (i.e., observer- and laboratory-based).

Conclusion. DAS28 is influenced by tender points, even in the non-fibromyalgia range, falsely suggesting higher disease activity and decreasing the sensitivity of the DAS28 criterion of low disease activity or remission. When applying DAS28-guided "tight control" or "treat-to-target" treatment strategies in RA, evaluation of not only the DAS28, but also its individual components along with a full joint and physical evaluation including assessment of TP is required to reliably estimate the individual's disease activity, which guides therapeutic decisions. (First Release Oct 15 2011; J Rheumatol 2012;39:22-7; doi:10.3899/jrheum.110072)
\end{abstract}

Key Indexing Terms:

RHEUMATOID ARTHRITIS DISEASE ACTIVITY 28-JOINT DISEASE ACTIVITY SCORE $\begin{array}{lll}\text { TIGHT CONTROL TREAT-TO-TARGET } & \text { TENDER POINTS FIBROMYALGIA }\end{array}$

In rheumatoid arthritis (RA), treatment strategies tailored to the individual patient to achieve a predefined level of low disease activity or remission are advocated, that is, "tight control" and "treat-to-target" strategies ${ }^{1,2}$. To this aim, generally the 28-joint Disease Activity Score (DAS28) is used ${ }^{3}$. It consists of 4 components: erythrocyte sedimentation rate (ESR),

From the Department of Rheumatology and Clinical Immunology, University Medical Center Utrecht, Utrecht; Sint Antonius Hospital, Nieuwegein; Meander Medical Center, Amersfoort; Diakonessen Hospital, Utrecht; and Hospital St. Jansdal, Harderwijk, The Netherlands.

E. Ton, MD, Rheumatologist; M.F. Bakker, MSc, PhD; S.M.M. Verstappen, MSc, PhD, Epidemiologist; J.W.J. Bijlsma, MD, PhD, Rheumatologist; J.W.G. Jacobs, MD, PhD, Rheumatologist, Department of Rheumatology and Clinical Immunology, University Medical Center Utrecht; E.J.ter Borg, MD, PhD, Rheumatologist, Sint Antonius Hospital; I.G.A. van Albada-Kuipers, MD, Rheumatologist, Meander Medical Center; Y. Schenk, MD, Rheumatologist, Diakonessen Hospital; M.J. van der Veen, MD, PhD, Rheumatologist, Hospital St. Jansdal.

Address correspondence to Dr.J.W.G. Jacobs, Department of Rheumatology and Clinical Immunology, University Medical Center Utrecht, Heidelberglaan 100, 3584 CX Utrecht, The Netherlands. E-mail:j.w.g.jacobs@umcutrecht.nl

Accepted for publication August 23, 2011.
28 tender joint count (TJC), 28 swollen joint count (SJC), and visual analog scale for general health (VAS-GH). In DAS28, the TJC has twice the contribution compared to that of the SJC (Figure 1). The DAS28 was developed and validated to evaluate disease activity status in groups of patients with RA participating in clinical trials, but has not been validated for use in the individual patient; the reliability of DAS28 for assessing disease activity in individual patients can be questioned ${ }^{4,5,6}$. Misclassification in low disease activity might be because the joints of ankles and feet are not included in the DAS28 ${ }^{6}$. Another cause of misclassification by the DAS28 is raised ESR due to reasons other than disease activity, e.g., low serum albumin, anemia, infection, or paraproteinemia. Further, in 2 recent studies DAS28 was reported to overestimate disease activity in patients with RA who also had fibromyalgia $(\mathrm{FM})^{7,8}$, which is the case in $12 \%-17 \%$ of patients with $\mathrm{RA}^{8,9,10,11,12}$.

The aim of our study was to determine whether tender points (TP) influence the DAS28, the individual components of the DAS28, and other disease variables in patients with RA. 


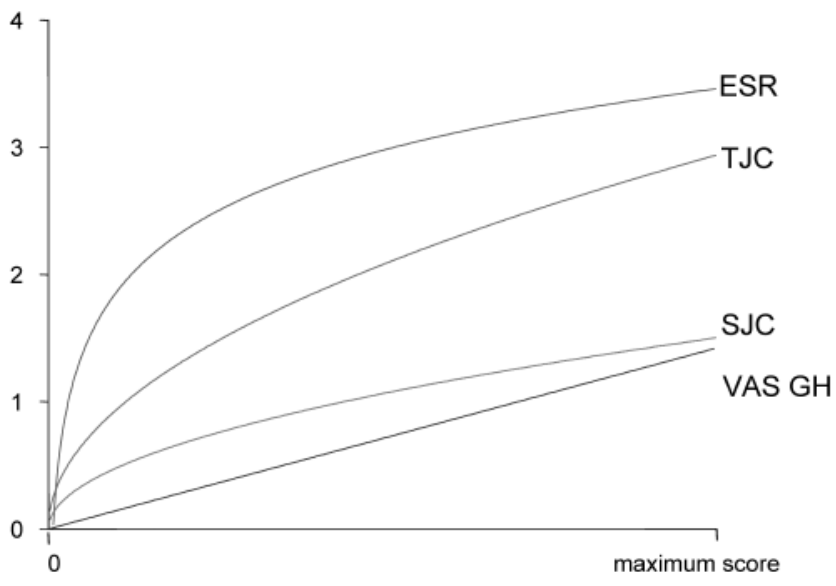

Figure 1. Contribution to the total 28-joint Disease Activity Score (DAS28) of the DAS28 individual components. Y-axis shows units of the DAS28; $\mathrm{x}$-axis: 0 to the maximum range of the plotted variables. TJC: tender joint count (0-28); SJC: swollen joint count (0-28); ESR: erythrocyte sedimentation rate $(0-140 \mathrm{~mm} / \mathrm{h})$; VAS-GH: visual analog scale for general health $(0-100 ; 100=$ worst score $)$. DAS28 $=0.56 \sqrt{ }($ TJC $)+0.28 \sqrt{ }($ SJC $)+0.70$ $\ln (\mathrm{ESR})+0.014$ VAS-GH.

\section{MATERIALS AND METHODS}

For this explorative study, 200 consecutive outpatients with RA seen in one university hospital by 3 rheumatologists and in 4 general hospitals by 6 rheumatologists, collaborating in the Utrecht Rheumatoid Arthritis Cohort study group, were included. All patients had established RA according to the 1987 classification criteria ${ }^{13}$ and all patients had given informed consent. They received antirheumatic medication according to treatment protocols used in all participating hospitals; $65 \%$ currently used methotrexate as single disease-modifying antirheumatic drug (DMARD), 7\% used hydroxychloroquine, $3 \%$ intramuscular gold, $2 \%$ azathioprine, $1 \%$ sulfasalazine, $0.5 \%$ cyclosporine, $0.5 \%$ leflunomide, and $0.5 \%$ oral gold; $10 \%$ used a combination of these DMARD and $10 \%$ currently used no DMARD. Two patients used anti-tumor necrosis factor- $\alpha$ drugs. During an outpatient clinic visit, ESR, TJC, SJC, and VAS-GH $(0-100 ; 100=$ worst score $)$ were assessed and the DAS28 was calculated ${ }^{3}$. In addition, tender point assessment according to the 1990 American College of Rheumatology (ACR) criteria for FM was performed ${ }^{14}$. Data for duration of early morning stiffness (0-180 minutes), VAS pain $(0-100$ scale; $0=$ no pain), and the Health Assessment Questionnaire (HAQ; 0-3 scale; $0=$ no disability) were obtained.

Statistical analyses. Descriptive statistics were used to summarize patient characteristics. Because of non-normal distribution of these data, median values are given with 10th-90th percentile values. The assessed 5-point scale for TP $(0=$ no pain to $4=$ pain plus flinch or withdrawal $)$ was recoded into a dichotomous score $(0=$ absence, $\geq 1=$ presence of TP). The sum of the dichotomous scores was the tender point count (TPC), range 0-18. Patients were categorized according to the TPC into 1 of 4 TPC classes: $0,1-5,6-10$, and $\geq 11$ TP. The cutoff of 1 was chosen because a major part of the population had no TP; the cutoff of 6 was based on the suggestion that, in clinical practice, for individuals a TPC $\geq 6$ might better discriminate patients with FM from those without $\mathrm{FM}^{15}$; and the cutoff of $\geq 11$ was based on the $1990 \mathrm{FM}$ classification criteria ${ }^{14}$. DAS28 and its individual components were analyzed in the different TP classes and tested for significant differences with Kruskal-Wallis and Mann-Whitney U tests. Spearman correlation coefficients were calculated for TPC, DAS28, and HAQ with patients' ages and disease duration and disease activity variables. A $p$ value $<0.05$ was considered statistically significant. Analyses were carried out using SPSS version 16 and NCSS 2007.

\section{RESULTS}

Of the 200 patients studied, 4 were excluded from analyses because of missing data, leaving 196 eligible for evaluation. Patient characteristics are summarized in Table 1. According to published criteria $^{3}, 49 \%$ of the patients had active RA defined as high (DAS28 $>5.1,8 \%$ of patients) or moderate (DAS28 $>3.2$ and $\leq 5.1,41 \%$ of patients) disease activity; $14 \%$ had low disease activity (DAS28 $\geq 2.6$ and $\leq 3.2$ ); and $37 \%$ were in remission (DAS28 $<2.6$ ). Among all patients, $43 \%$ had no TP, $30 \%$ had a TPC of $1-5,12 \%$ (15\% of women vs $3 \%$ of men) had TPC of $6-10$, and $15 \%$ (17\% of women vs $8 \%$ of men) had TPC $\geq 11$ (Table 1). Overall, women had significantly more TP than men: median 2 versus 0 , respectively $(\mathrm{p}<0.005)$. The distribution of TP and joint counts according to 4 regions of the body - upper and lower and left and right extremities - is illustrated in Figure 2.

Figure 3 shows that in the TPC classes, with increasing TPC, the median DAS28, TJC, and VAS-GH increased significantly; in contrast, the ESR and SJC were not statistically significantly different among the 4 groups (Table 1). Similarly, the DAS28, TJC, and VAS-GH correlated significantly with TPC, in contrast to SJC and ESR; the TPC also correlated significantly with early morning stiffness, VAS pain, and the HAQ results (Table 2).

Among patients with active RA according to DAS28 $>3.2$, $13 \%$ had no swollen joint of the 28 joints assessed, indicating misclassification.

\section{DISCUSSION}

The DAS28 is a widely used instrument for assessing disease activity in patients with RA. Reliability of DAS28 in the individual patient can be questioned, especially if there is concomitant FM, as shown in other studies ${ }^{7,8}$, or if there are tender points, even in the non-FM range, as shown in our study. Although the increase in the median DAS28 of 2.6 in the group without TP to 3.3 in the group with 1-5 TP (Table 2) might not seem impressive on first consideration, it was statistically significant, and one should keep in mind that DAS28 2.6 is the cutoff for remission and DAS 3.2 that for moderate disease activity. In the group with 1-5 TP compared to the group with 6-10 TP, the median DAS28 scores were 3.3 and 3.4, but mean DAS28 3.3 and 3.6, respectively (Table 1). With increasing TPC, the less objective DAS28 components (i.e., based on patient report: the VAS for general well-being and TJC) showed increasingly higher scores, but the more objective DAS28 components (i.e., observer- and laboratory-based SJC and ESR) did not. This is in accord with a previous study in which patients with FM - without RA and with normal ESR values and no swollen joints - had high scores on the DAS28 ${ }^{16}$. Similarly to our study, in another study in patients with both RA and FM, statistically significant associations were found between TPC and DAS28 but not between TPC and ESR; in contrast to our findings, there was also a significant correlation between TPC and SJC ${ }^{12}$. 
Table 1. Patient characteristics and clinical assessments of total study population and subgroups according to tender point classes. All values are median (10th to 90 th percentile), unless otherwise indicated.

\begin{tabular}{|c|c|c|c|c|c|c|}
\hline Characteristic & $\begin{array}{c}\text { Total } \\
\mathrm{n}=196(100 \%)\end{array}$ & $\begin{array}{c}\text { TPC 0, } \\
\mathrm{n}=84(43 \%)\end{array}$ & $\begin{array}{c}\text { TPC } 1-5, \\
\mathrm{n}=60(30 \%)\end{array}$ & $\begin{array}{c}\text { TPC 6-10, } \\
\mathrm{n}=23(12 \%)\end{array}$ & $\begin{array}{c}\text { TPC } \geq 11, \\
n=29(15 \%)\end{array}$ & $\mathrm{p}^{*}$ \\
\hline Age, yrs & $59(40-78)$ & $58(37-76)$ & $58(42-76)$ & $60(43-83)$ & $61(43-79)$ & NS \\
\hline Female, \% & 70 & 78 & 73 & 91 & 83 & $<0.005$ \\
\hline Disease duration, yrs & $4(1-8)$ & $4(1-8)$ & $2(1-8)$ & $5(1-9)$ & $5(1-8)$ & NS \\
\hline RF-positive, $\%$ & 66 & 66 & 70 & 61 & 66 & NS \\
\hline EMS & $5(0-90)$ & $0(0-30)$ & $15(0-60)$ & $20(0-120)$ & $18(0-285)$ & $<0.00001$ \\
\hline VAS pain & $14(0-66)$ & $5(0-50)$ & $12(0-68)$ & $36(8-84)$ & $33(3-79)$ & $<0.00000$ \\
\hline HAQ & $1(0-2)$ & $0.5(0-1.6)$ & $1.0(0.1-2.2)$ & $1.6(1.1-2.3)$ & $1.5(0.2-2.4)$ & $<0.00000$ \\
\hline $\mathrm{TPC}$ & $1(0-13)$ & $0(0-0)$ & $2(1-5)$ & $8(6-10)$ & $16(12-18)$ & NA \\
\hline DAS28 & $3.1(1.3-5.1)$ & $2.6(1.2-4.7)$ & $3.3(1.5-5.1)^{* * *}$ & $3.4(1.9-5.1)^{* *}$ & $4.1(2.3-5.8)^{* *}$ & $<0.001$ \\
\hline TJC & $1(0-10)$ & $0(0-5)$ & $2(0-7)$ & $2(0-17)$ & $5(0-19)$ & $<0.0001$ \\
\hline SJC & $1(0-7)$ & $1(0-8)$ & $1(0-7)$ & $1(0-11)$ & $1(0-8)$ & NS \\
\hline $\mathrm{ESR}, \mathrm{mm} / \mathrm{h}$ & $15(4-41)$ & $12(3-42)$ & $19(4-49)$ & $19(2-36)$ & $15(5-42)$ & NS \\
\hline VAS-GH, mm & $26(1-67)$ & $18(0-60)$ & $29(3-70)$ & $34(9-79)$ & $42(10-70)$ & $<0.01$ \\
\hline
\end{tabular}

* Kruskal-Wallis test. ** All statistically significantly higher compared to DAS28 in group with TPC = 0 (Mann-Whitney U tests); corresponding means (SD) of DAS28 respectively 2.9 (1.4), 3.3 (1.3), 3.6 (1.6), and 4.0 (1.2); p < 0.0004 in ANOVA. RF: rheumatoid factor; EMS: early morning stiffness (0-180 min); VAS pain: visual analog scale for pain (0-100; $0=$ no pain); HAQ: Health Assessment Questionnaire (0-3; $0=$ no disability); TPC: tender point count $(0-18)$; DAS28: Disease Activity Score; TJC: tender joint count (0-28); SJC: swollen joint count (0-28); ESR: erythrocyte sedimentation rate (0-140 mm/h); VAS-GH: VAS for general health $(0-100 \mathrm{~mm}, 100=$ worst score $)$; NS: not statistically significant; NA: not applicable.

These findings reduce the sensitivity of DAS28 to assess low disease activity or remission in individuals (more false-negative cases). We did not investigate this, but other causes of a raised ESR than disease activity (low serum albumin, anemia, or a paraprotein) would have the same effect. In the DAS28, the absence of joints of the feet, which are frequently involved in $\mathrm{RA}^{17}$, reduces the specificity of the DAS28 for this aim (more false-positive cases) ${ }^{6}$.

To improve the specificity of assessing remission in patients using individual DAS28-guided "tight control" and "treat-to-target" strategies, one could add to the DAS28 criterion of remission the criterion of absence of any swollen joint. This would mean assessment of all joints frequently involved in RA. If the presence or absence of arthritis cannot be assessed reliably, ultrasonography could be applied ${ }^{18}$. To improve the sensitivity of the DAS28 to assess low disease activity or remission in individuals, other influences falsely increasing the DAS28 should be taken into account, such as TP and elevations of the ESR not specific for RA. This would mean looking not only at the DAS28, but also at its individual components.

In contrast to the prevalence of FM, the prevalence of TP in the non-FM range in patients with RA using antirheumatic medication (including nonsteroidal antiinflammatory drugs and analgesics) is not known. A substantial part of our population had no TP. Our study comprised a population-based RA cohort visiting academic and general rheumatology outpatient clinics on a regular basis. In The Netherlands, virtually all patients with RA are treated by rheumatologists, not by general practitioners. Thus our sample reflects a common RA population.

One could speculate on the pathophysiological mecha- nisms of the origin of TP in RA. Our findings that ESR and SJC are not associated with TPC refute a direct causative relation of joint inflammation and TP. It is possible that physical deconditioning induced by less physical activity in the past because of signs and symptoms of RA plays an indirect role. Also, other mechanisms could influence pain and tenderness in a patient with RA, e.g., joint destruction, central amplification mechanisms, and sleep disorders.

The prevalence of concomitant FM in our study population was not known, but the number of patients meeting the cutoff of $11 \mathrm{TP}$ according to the 1990 criteria for FM was 15\% (Table 2), and this is consistent with the published prevalence of secondary FM of 12\%-17\% among patients with $\mathrm{RA}^{8,9,10,11,12}$. We chose to assess TP but not to apply the ACR 1990 criteria for FM, as the FM 1990 criterion of chronic generalized pain is difficult to interpret in patients with RA. As the 1990 ACR criteria for FM are intended for classifying groups, especially for research, their relevance in clinical practice for individuals has been questioned. It has been suggested that in clinical practice for individuals, a TPC $\geq 6$ might discriminate better between patients with FM and those without $\mathrm{FM}^{15}$. In our study, this TPC would also influence the reliability of the DAS28. New criteria sets have been developed not only for FM and RA, but also for RA remis$\operatorname{sion}^{19,20,21}$; the new Boolean criteria of remission permit only 1 swollen joint to be present. In our view this is a real improvement compared to DAS28 remission, because in RA patients with DAS28 remission it is not infrequently the case that 5-10 swollen joints are present ${ }^{6}$.

The DAS28 disease activity index is influenced by coexistence of tender points, even in the non-FM range, due to the clear association of the TPC with the less objective DAS28 Personal non-commercial use only. The Journal of Rheumatology Copyright @ 2012 . All rights reserved. 


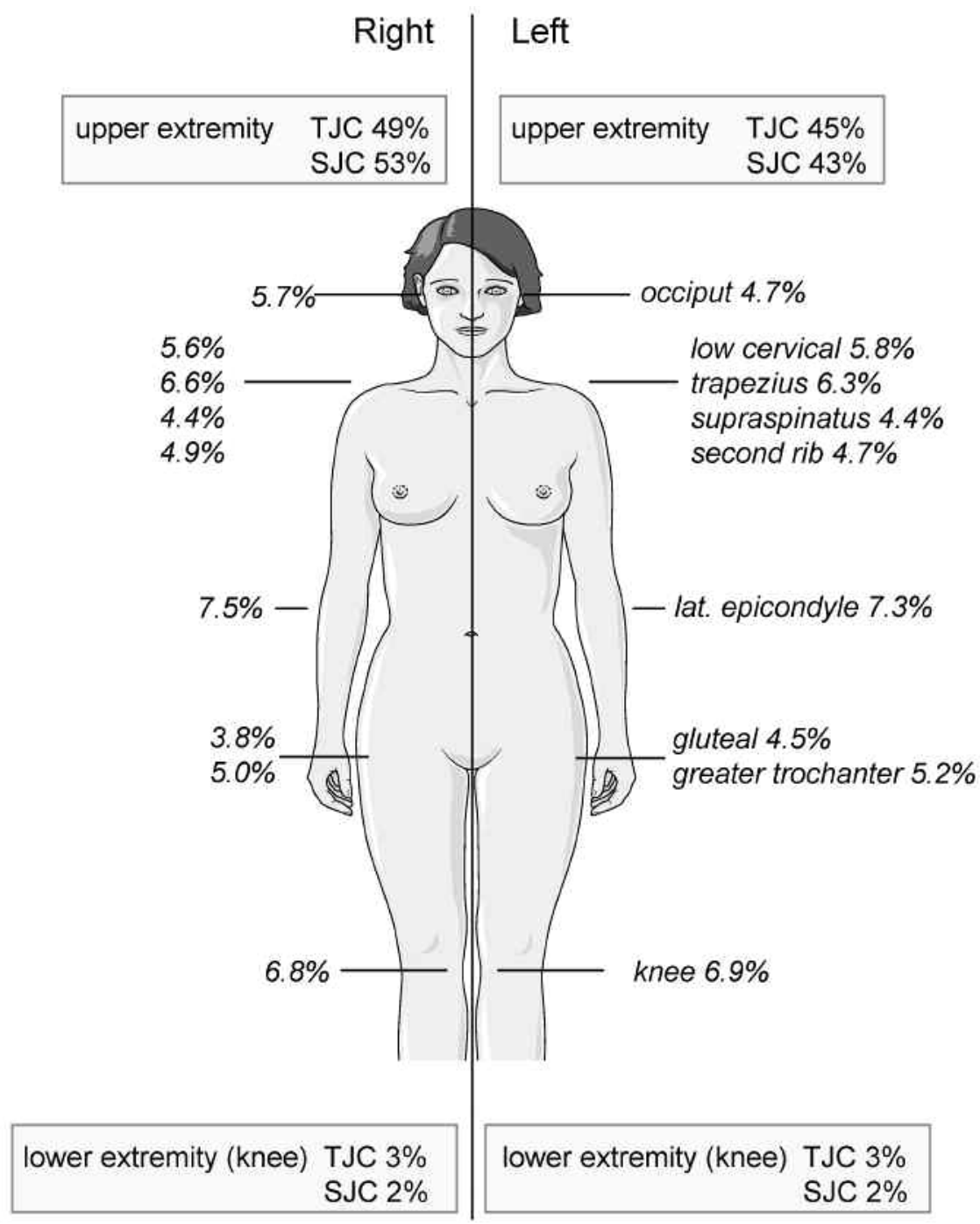

Figure 2. Distribution of tender points and joint counts. Mean prevalences of individual tender points shown as percentages of mean total tender point count in italic. Mean prevalences of tender joints for the upper and lower and right and left body regions separately are shown in the boxes as percentages of mean total tender joint count (TJC); similarly, prevalences of swollen joints are shown as percentages of mean total swollen joint count (SJC). TJC and SJC are part of DAS28 (assessing 28 joints), of which the only joint that is assessed at the lower extremity is the knee.

components VAS-GH and TJC. When applying DAS28 for "treat-to-target" treatment strategies, evaluation of not only the DAS28 but also its individual components along with a full physical evaluation, according to good clinical practice, including assessment of all joints frequently involved in RA (and also ankles and feet) and of TP is required for adequate estimation of the individual's disease activity, and for making appropriate therapeutic decisions.

\section{REFERENCES}

1. Smolen JS, Landewé R, Breedveld FC, Dougados M, Emery P, Gaujoux-Viala C, et al. EULAR recommendations for the management of rheumatoid arthritis with synthetic and biological disease-modifying antirheumatic drugs. Ann Rheum Dis 2010;69:964-75.
2. Bakker MF, Jacobs JW, Verstappen SM, Bijlsma JW. Tight control in the treatment of rheumatoid arthritis: efficacy and feasibility. Ann Rheum Dis 2007;66 Suppl 3:iii56-60.

3. Prevoo ML, van 't Hof MA, Kuper HH, van Leeuwen MA, van de Putte LB, van Riel PL. Modified disease activity scores that include twenty-eight-joint counts. Development and validation in a prospective longitudinal study of patients with rheumatoid arthritis. Arthritis Rheum 1995;38:44-8.

4. Taylor WJ, Harrison AA, Highton J, Chapman P, Stamp L, Dockerty J, et al. Disease Activity Score 28-ESR bears a similar relationship to treatment decisions across different rheumatologists, but misclassification is too frequent to replace physician judgement. Rheumatology 2008;47:514-8.

5. Wolfe F, Michaud K, Pincus T, Furst D, Keystone E. The Disease Activity Score is not suitable as the sole criterion for initiation and evaluation of anti-tumor necrosis factor therapy in the clinic: Discordance between assessment measures and limitations in 


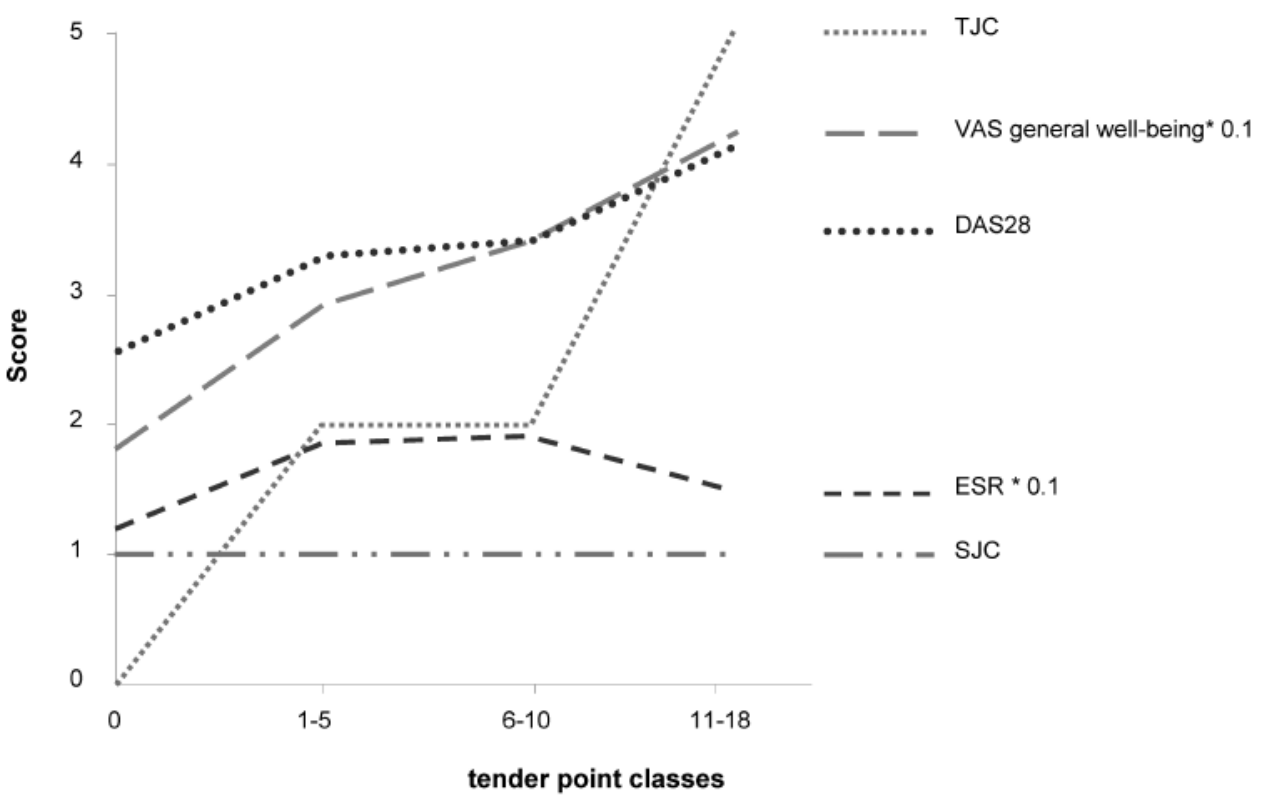

Figure 3. Medians of DAS28 and its individual components in the 4 tender point classes. TPC $0, \mathrm{n}=84$; TPC $1-5, n=60$; TPC $6-11, n=23$; TPC $11-18, n=29$ patients. TPC: tender point count $(0-18)$; TJC: tender joint count (0-28); SJC: swollen joint count (0-28); ESR: erythrocyte sedimentation rate $(0-140 \mathrm{~mm} / \mathrm{h}$ ); VAS-GH: visual analog scale for general health $(0-100 ; 100=$ worst score $)$.

Table 2. Correlation coefficients (Spearman's rho) of TPC, DAS28, and HAQ with patients' ages and disease duration and disease activity variables.

\begin{tabular}{llll}
\hline Variables & TPC & DAS28 & HAQ \\
\hline Age & 0.05 & -0.07 & $0.16^{*}$ \\
Disease duration & 0.01 & $-0.18^{*}$ & 0.05 \\
EMS & $0.42^{* *}$ & $0.50^{* *}$ & $0.51^{* *}$ \\
VAS pain & $0.41^{* *}$ & $0.54^{* *}$ & $0.57^{* *}$ \\
HAQ & $0.48^{* *}$ & $0.52^{* *}$ & - \\
TPC & - & $0.35^{* *}$ & $0.48^{* *}$ \\
DAS28 & $0.35^{* *}$ & - & $0.52^{* *}$ \\
TJC & $0.37^{* *}$ & ND & $0.42^{* *}$ \\
SJC & 0.08 & ND & $0.17^{*}$ \\
ESR & 0.14 & ND & $0.27^{* *}$ \\
VAS-GH & $0.29^{* *}$ & ND & $0.58^{* *}$ \\
\hline
\end{tabular}

$* \mathrm{p}<0.05 ; * * \mathrm{p}<0.0001$. ND: not done: components of DAS28. TPC: tender point count (0-18); EMS: early morning stiffness (0-180 min); DAS28: Disease Activity Score assessing 28 joints; TJC: tender joint count (0-28); SJC: swollen joint count (0-28); ESR: erythrocyte sedimentation rate $(0-140 \mathrm{~mm} / \mathrm{h})$; VAS-GH: visual analog scale for general health $(0-100 ; 0=$ very good); VAS pain: visual analog scale for pain $(0-100$; $0=$ no pain); HAQ: Health Assessment Questionnaire $(0-3 ; 0=$ no disability).

questionnaire use for regulatory purposes. Arthritis Rheum 2005;52:3873-9.

6. Landewé R, van der Heijde D, van der Linden S, Boers M. Twenty-eight-joint counts invalidate the DAS28 remission definition owing to the omission of the lower extremity joints: A comparison with the original DAS remission. Ann Rheum Dis 2006;65:637-41.

7. Coury F, Rossat A, Tebib A, Letroublon MC, Gagnard A, Fantino $\mathrm{B}$, et al. Rheumatoid arthritis and fibromyalgia: A frequent unrelated association complicating disease management. J Rheumatol 2009;36:58-62.

8. Ranzolin A, Brenol JC, Bredemeier M, Guarienti J, Rizzatti M, Feldman D, et al. Association of concomitant fibromyalgia with worse Disease Activity Score in 28 joints, Health Assessment Questionnaire, and Short Form 36 scores in patients with rheumatoid arthritis. Arthritis Rheum 2009;61:794-800.

9. Naranjo A, Ojeda S, Francisco F, Erausquin C, Rua-Figueroa I, Rodriguez-Lozano C. Fibromyalgia in patients with rheumatoid arthritis is associated with higher scores of disability. Ann Rheum Dis 2002;61:660-1.

10. Wolfe F, Michaud K. Severe rheumatoid arthritis (RA), worse outcomes, comorbid illness, and sociodemographic disadvantage characterize RA patients with fibromyalgia. J Rheumatol 2004;31:695-700.

11. Wolfe F, Cathey MA, Kleinheksel SM. Fibrositis (fibromyalgia) in rheumatoid arthritis. J Rheumatol 1984;11:814-8.

12. Dhir V, Lawrence A, Aggarwal A, Misra R. Fibromyalgia is common and adversely affects pain and fatigue perception in North Indian patients with rheumatoid arthritis. J Rheumatol 2009;36:2443-8.

13. Arnett FC, Edworthy SM, Bloch DA, McShane DJ, Fries JF, Cooper NS, et al. The American Rheumatism Association 1987 revised criteria for the classification of rheumatoid arthritis. Arthritis Rheum 1988;31:315-24.

14. Wolfe F, Smythe HA, Yunus MB, Bennett RM, Bombardier C, Goldenberg DL, et al. The American College of Rheumatology 1990 criteria for the classification of fibromyalgia. Report of the Multicenter Criteria Committee. Arthritis Rheum 1990;33:160-72.

15. Katz RS, Wolfe F, Michaud K. Fibromyalgia diagnosis: A comparison of clinical, survey, and American College of Rheumatology criteria. Arthritis Rheum 2006;54:169-76.

16. Leeb BF, Andel I, Sautner J, Nothnagl T, Rintelen B. The DAS28 in rheumatoid arthritis and fibromyalgia patients. Rheumatology 2004;43:1504-7.

17. Hulsmans HM, Jacobs JW, van der Heijde DM, van Personal non-commercial use only. The Journal of Rheumatology Copyright (c) 2012. All rights reserved. 
Albada-Kuipers GA, Schenk Y, Bijlsma JW. The course of radiologic damage during the first six years of rheumatoid arthritis. Arthritis Rheum 2000;43:1927-40.

18. Cheung PP, Dougados M, Gossec L. Reliability of ultrasonography to detect synovitis in rheumatoid arthritis: A systematic literature review of 35 studies (1,415 patients). Arthritis Care Res 2010;62:323-34.

19. Wolfe F, Clauw DJ, Fitzcharles MA, Goldenberg DL, Katz RS, Mease P, et al. The American College of Rheumatology preliminary diagnostic criteria for fibromyalgia and measurement of symptom severity. Arthritis Care Res 2010;62:600-10.
20. Aletaha D, Neogi T, Silman AJ, Funovits J, Felson DT, Bingham CO 3rd, et al. 2010 rheumatoid arthritis classification criteria: an American College of Rheumatology/European League Against Rheumatism collaborative initiative. Ann Rheum Dis 2010;69:1580-8.

21. Felson DT, Smolen JS, Wells G, Zhang B, van Tuyl LH, Funovits J, et al. American College of Rheumatology / European League against Rheumatism provisional definition of remission in rheumatoid arthritis for clinical trials. Ann Rheum Dis 2011;70:404-13. 